Louisiana State University

LSU Digital Commons

$5-14-2019$

\title{
Probing the interplay between geometric and electronic-structure features via high-harmonic spectroscopy
}

\author{
T. T. Gorman \\ The Ohio State University \\ T. D. Scarborough \\ The Ohio State University \\ P. M. Abanador \\ Louisiana State University \\ F. Mauger \\ Louisiana State University \\ D. Kiesewetter \\ The Ohio State University
}

See next page for additional authors

Follow this and additional works at: https://digitalcommons.Isu.edu/chemistry_pubs

\section{Recommended Citation}

Gorman, T., Scarborough, T., Abanador, P., Mauger, F., Kiesewetter, D., Sándor, P., Khatri, S., Lopata, K., Schafer, K., Agostini, P., Gaarde, M., \& Dimauro, L. (2019). Probing the interplay between geometric and electronic-structure features via high-harmonic spectroscopy. Journal of Chemical Physics, 150 (18) https://doi.org/10.1063/1.5086036

This Article is brought to you for free and open access by the Department of Chemistry at LSU Digital Commons. It has been accepted for inclusion in Faculty Publications by an authorized administrator of LSU Digital Commons. For more information, please contact ir@lsu.edu. 


\section{Authors}

T. T. Gorman, T. D. Scarborough, P. M. Abanador, F. Mauger, D. Kiesewetter, P. Sándor, S. Khatri, K. Lopata, K. J. Schafer, P. Agostini, M. B. Gaarde, and L. F. Dimauro 
Louisiana State University

LSU Digital Commons

$5-14-2019$

\title{
Probing the interplay between geometric and electronic-structure features via high-harmonic spectroscopy
}

\author{
T. T. Gorman \\ The Ohio State University \\ T. D. Scarborough \\ The Ohio State University \\ P. M. Abanador \\ Louisiana State University \\ F. Mauger \\ Louisiana State University \\ D. Kiesewetter \\ The Ohio State University
}

See next page for additional authors

Follow this and additional works at: https://digitalcommons.Isu.edu/physics_astronomy_pubs

\section{Recommended Citation}

Gorman, T., Scarborough, T., Abanador, P., Mauger, F., Kiesewetter, D., Sándor, P., Khatri, S., Lopata, K., Schafer, K., Agostini, P., Gaarde, M., \& Dimauro, L. (2019). Probing the interplay between geometric and electronic-structure features via high-harmonic spectroscopy. Journal of Chemical Physics, 150 (18) https://doi.org/10.1063/1.5086036

This Article is brought to you for free and open access by the Department of Physics \& Astronomy at LSU Digital Commons. It has been accepted for inclusion in Faculty Publications by an authorized administrator of LSU Digital Commons. For more information, please contact ir@lsu.edu. 


\section{Authors}

T. T. Gorman, T. D. Scarborough, P. M. Abanador, F. Mauger, D. Kiesewetter, P. Sándor, S. Khatri, K. Lopata, K. J. Schafer, P. Agostini, M. B. Gaarde, and L. F. Dimauro 


\section{Probing the interplay between geometric and electronic-structure features via high- harmonic spectroscopy}

Cite as: J. Chem. Phys. 150, 184308 (2019); https://doi.org/10.1063/1.5086036

Submitted: 17 December 2018 . Accepted: 17 April 2019. Published Online: 14 May 2019

T. T. Gorman, T. D. Scarborough (D), P. M. Abanador (D), F. Mauger (D), D. Kiesewetter, P. Sándor (D), S. Khatri, K. Lopata (D) K. J. Schafer, P. Agostini, M. B. Gaarde, and L. F. DiMauro

\section{COLLECTIONS}

Paper published as part of the special topic on Ultrafast molecular sciences by femtosecond photons and electrons

Note: The paper is part of the JCP Special Topic on Ultrafast molecular sciences by femtosecond photons and electrons.
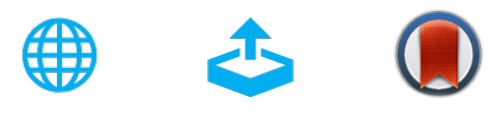

View Online

Export Citation

\section{ARTICLES YOU MAY BE INTERESTED IN}

High-harmonic spectroscopy of transient two-center interference calculated with timedependent density-functional theory

Structural Dynamics 6, 044101 (2019); https://doi.org/10.1063/1.5111349

Ultrafast photodissociation dynamics and nonadiabatic coupling between excited electronic states of methanol probed by time-resolved photoelectron spectroscopy The Journal of Chemical Physics 150, 114301 (2019); https://doi.org/10.1063/1.5079549 Angle-dependent strong-field ionization of halomethanes

The Journal of Chemical Physics 151, 194308 (2019); https://doi.org/10.1063/1.5121711
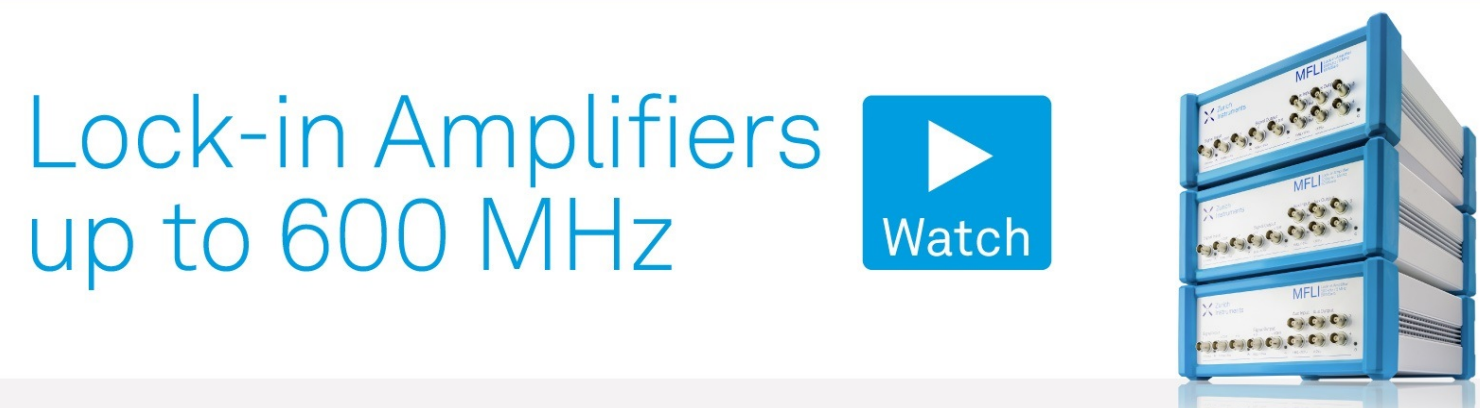


\title{
Probing the interplay between geometric and electronic-structure features via high-harmonic spectroscopy
}

\author{
Cite as: J. Chem. Phys. 150, 184308 (2019); doi: 10.1063/1.5086036 \\ Submitted: 17 December 2018 - Accepted: 17 April 2019 • \\ Published Online: 14 May 2019
}

\begin{abstract}
T. T. Gorman, ' T. D. Scarborough, (D) P. M. Abanador, ${ }^{2}$ (D) F. Mauger, ${ }^{2}$ (D) D. Kiesewetter, ${ }^{1}$ P. Sándor, ${ }^{3}$
S. Khatri, ${ }^{3}$ K. Lopata, ${ }^{4}$ (D) K. Schafer, ${ }^{2}$ P. Agostini, ${ }^{1}$ M. B. Gaarde, ${ }^{2}$ and L. F. DiMauro'
\end{abstract}

\begin{abstract}
AFFILIATIONS
${ }^{1}$ Department of Physics, The Ohio State University, Columbus, Ohio 43210, USA

${ }^{2}$ Department of Physics and Astronomy, Louisiana State University, Baton Rouge, Louisiana 70803, USA

${ }^{3}$ Department of Physics, University of Virginia, Charlottesville, Virginia 22904, USA

${ }^{4}$ Department of Chemistry, Louisiana State University, Baton Rouge, Louisiana 70803, USA
\end{abstract}

Note: The paper is part of the JCP Special Topic on Ultrafast molecular sciences by femtosecond photons and electrons.

\begin{abstract}
We present molecular-frame measurements of the recombination dipole matrix element (RDME) in $\mathrm{CO}_{2}, \mathrm{~N}_{2} \mathrm{O}$, and carbonyl sulfide (OCS) molecules using high-harmonic spectroscopy. Both the amplitudes and phases of the RDMEs exhibit clear imprints of a two-center interference minimum, which moves in energy with the molecular alignment angle relative to the laser polarization. We find that whereas the angle dependence of this minimum is consistent with the molecular geometry in $\mathrm{CO}_{2}$ and $\mathrm{N}_{2} \mathrm{O}$, it behaves very differently in OCS; in particular, the phase shift which accompanies the two-center minimum changes sign for different alignment angles. Our results suggest that two interfering structural features contribute to the OCS RDME, namely, (i) the geometrical two-center minimum and (ii) a Cooper-like, electronic-structure minimum associated with the sulfur end of the molecule. We compare our results to ab initio calculations using time-dependent density functional theory and present an empirical model that captures both the two-center and the Cooper-like interferences. We also show that the yield from unaligned samples of two-center molecules is, in general, reduced at high photon energies compared to aligned samples, due to the destructive interference between molecules with different alignments.
\end{abstract}

Published under license by AIP Publishing. https://doi.org/10.1063/1.5086036

\section{INTRODUCTION}

Recent years have seen rapid development in the use of highharmonic spectroscopy (HHS) as a means to study molecular structure and dynamics at the space and time scales of the electron. ${ }^{1-6}$ HHS relies on the process of high-harmonic generation (HHG) in which a natural attosecond time scale is defined by the periodic motion of an electron in a molecule through tunnel ionization and subsequent recombination in each half cycle of a driving infrared/optical field. ${ }^{7-10}$ Based on the idea that both molecular structure and dynamics may render the inverse-photoionization process of recombination time- and space-dependent, HHS attempts to characterize the complex recombination dipole matrix element (RDME) via detailed measurement of the spectral properties of the emitted extreme ultraviolet (XUV) radiation. ${ }^{3,11,12}$ While it is only the combined measurement of both the spectral intensity and phase, in the molecular frame, which can fully characterize the RDME, most HHS studies have characterized only the spectral intensity, with only a few also including the spectral phase measurements.

A purely structural effect that can be characterized using HHS, and that manifests in both the spectral intensity and phase, is the socalled two-center interference (TCI). TCI occurs in small molecules where the highest-occupied molecular orbital (HOMO) is predominantly composed of two centers of charge density. The spectral minimum that results from destructive interference between recombination to both sites has been experimentally and theoretically studied in a number of systems. ${ }^{1,2,4,16-18}$ Conceptually, thinking of the 
recolliding electron as a plane wave scattering on either center, the TCI condition reads ${ }^{19}$

$$
k_{e} R \cos \theta+\Delta \Phi\left(k_{e}, \theta\right)=(2 m+1) \pi
$$

for any integer $m$, as illustrated in Fig. 1 . Here, $k_{e}=2 \pi / \lambda_{e}$ is the electron wave number, $\lambda_{e}$ is the de Broglie wavelength, $R$ is the effective distance between the two centers of electron density (the lobes in Fig. 1), and $\theta$ is the rescattering angle with respect to the molecular axis. The structural contribution $\Delta \Phi$ is constant $(\pi)$ for the symmetric $\mathrm{CO}_{2}$ molecule where the two charge centers have opposite phase, and its variation with energy and angle accounts for asymmetric contributions, e.g., due to an imbalance between the two centers like in carbonyl sulfide (OCS), or the effect of a permanent dipole moment like in $\mathrm{N}_{2} \mathrm{O} .{ }^{20}$ Although Eq. (1) is only approximate because additional terms as well as amplitude variations can yield additional contributions to the RDME, it provides a useful qualitative approach to the behavior of the TCI minimum. In particular, for symmetric molecules, Eq. (1) predicts the "geometrical" expectation for the location of the TCI minimum. Because the TCI minimum relies heavily on the destructive interference from the two centers, it is a highly sensitive probe of the RDME in the vicinity of the minimum.

For a molecule in which $\Delta \Phi\left(k_{e}, \theta\right)$ is nontrivial, however, the interplay between the geometric $k_{e} R \cos (\theta)$ term and the $\Delta \Phi\left(k_{e}, \theta\right)$ term is more complex. One such molecule is OCS. The HOMO of OCS can be thought of, in the linear combination of atomic orbitals (LCAO) representation, as the combination of a sulfur $3 p$ orbital and an oxygen $2 \mathrm{p}$ orbital. ${ }^{21}$ It is known that molecules that retain atomic $3 p$ character, such as sulfur- and chlorine-containing molecules, demonstrate features analogous to a Cooper minimum, ${ }^{15,22-24}$ i.e., a minimum in the spectral intensity accompanied by a spectral phase jump. Indeed, it has been shown that the photoionization cross section of the OCS HOMO drops significantly above $35 \mathrm{eV}$ and stays low until at least $100 \mathrm{eV}{ }^{25}$ The only previous HHS study of OCS was unable to characterize this phenomenon due to their reduced harmonic energy cutoff at near-infrared (NIR) wavelengths and the very small absorption cross section of OCS above $35 \mathrm{eV}$.

In this article, we present multidimensional, molecular-frame measurements of the spectral intensity and group delay (GD) of TCI

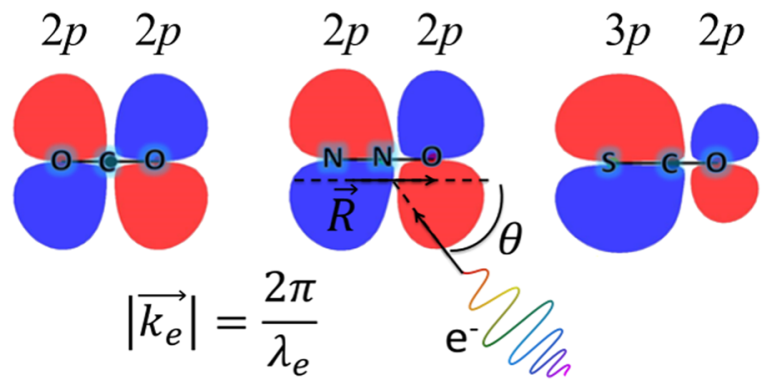

FIG. 1. Molecular two-center interference (TCI) occurs when an electron with de Broglie wavelength $\lambda_{e}$ is incident on a molecule with lobe separation $R$ and satisfies the criterion of Eq. (1). Isosurfaces of the HOMO orbitals for each molecule are represented with red and blue indicating opposite phases, together with their dominating LCAO composition. in $\mathrm{CO}_{2}, \mathrm{~N}_{2} \mathrm{O}$, and OCS, over a broad range of energies, using tunable midinfrared (MIR) drivers for HHG and impulsive molecular alignment to fix the molecular frame. We compare the experimental measurements to results of time-dependent density functional theory (TDDFT) simulations for $\mathrm{CO}_{2}$ and OCS and interpret our results using a conceptual model that includes both TCI and Cooperlike features. We demonstrate that the OCS spectral intensity and GD features in the vicinity of the TCI minima differ significantly from those of $\mathrm{CO}_{2}$ and $\mathrm{N}_{2} \mathrm{O}$. In particular, whereas $\mathrm{CO}_{2}$ and $\mathrm{N}_{2} \mathrm{O}$ exhibit primarily geometric TCIs, OCS also exhibits clear signs of the Cooper-like feature which adds to and interferes with the geometric contribution. We also show that the harmonic yields from unaligned samples of all three molecules are reduced at high energies compared to the aligned samples. We interpret this general feature in terms of destructive interference between the signals from molecules at different angles which have a TCI phase jump in different energy regions.

The calculations in this paper represent the first large-scale exploration of TDDFT as a means to identify structural features in molecular HHG, in particular, through the combination of amplitude and phase studies. ${ }^{27-30}$ We find, in general, that the TDDFT calculations capture the geometric TCI feature well but not the Cooperlike feature in OCS, the location of which is extremely sensitive to the details of the short-range molecular potential. ${ }^{31}$ TDDFT is among the few $a b$ initio methods that can handle the full subcycle dynamics of a multielectron system exposed to a strong field (for others, see Refs. 32 and 33) and represents a different approach to HHS calculations than those based on stepwise combinations of ionization, electron dynamics, and rescattering. ${ }^{3,5,12,34-37}$ Recent work showing that TDDFT gives a good description of attosecond charge migration as long as the initial condition is well defined, ${ }^{38,39}$ combined with our finding here that TDDFT recovers the geometric TCI features, bodes well for TDDFT as a potential tool to explore charge migration through HHS.

This article is structured as follows: Section II details the experimental apparatus and procedures used to measure HHG spectral intensities and GD. Additionally, this section describes the TDDFT methods and a physical model used to explain the experimental observations. Section III describes our experimental and theoretical results and their interpretations. Finally, we summarize our results in Sec. IV.

\section{METHODS}

\section{A. Experimental methods}

In these measurements, we utilize a commercial tunable, $1 \mathrm{kHz}$, MIR optical parametric amplifier (OPA) (HE-TOPAS by Light Conversion), pumped by a $785 \mathrm{~nm}, 55 \mathrm{fs}$ pump pulse. We use this OPA for two purposes. First, we use the MIR OPA to generate 75 fs pulses centered around $1300 \mathrm{~nm}$, each with $1 \mathrm{~mJ}$ of pulse energy for HHG. Second, we employ the depleted pump, which has $2 \mathrm{~mJ}$ of available pulse energy after the OPA, for molecular alignment. Using MIR wavelengths for HHG produces an extended cutoff when compared to the previous NIR measurements, ${ }^{26}$ critical for these low ionization potential $(I P)$ molecules where ground state depletion limits the maximum driving intensity. Additionally, longer driving wavelengths provide finer sampling in our GD measurements. Using the 
depleted pump for molecular alignment allows us to keep nearly constant alignment conditions while tuning the HHG wavelength, important for our GD measurement method to be described shortly. The power and spot sizes of each beam can be adjusted with variable apertures.

The measurement apparatus consists of a Mach-Zehnder interferometer and a $1 \mathrm{~m}$ magnetic bottle electron spectrometer (MBES), all of which is entirely contained in vacuum. The majority of the MIR is split to be focused with an $\mathrm{f}=400 \mathrm{~mm}$ focal mirror into the HHG gas source, leading to an intensity of approximately $(1 \pm 0.2) \times 10^{14} \mathrm{~W} / \mathrm{cm}^{2}$ for all molecules. A small portion of the MIR driver is retained to be recombined with the XUV light. Due to the low vapor pressure of OCS, we seed it at $10 \%$ in a helium buffer gas in order to minimize the effects of clustering while also achieving gas densities required for proper phase-matching. Doing so requires the use of a 200- $\mu \mathrm{m}$-nozzle-diameter Even-Lavie pulsed gas valve. Backing pressures between 15 and 20 bars were used with opening times between 23 and $30 \mu$ s, depending on dayto-day pulsed valve operation. We have confirmed that no significant XUV light is generated from the helium carrier gas, by testing a neat (undiluted) helium sample under the same generating conditions. $\mathrm{CO}_{2}$ and $\mathrm{N}_{2} \mathrm{O}$, however, are delivered neat and with a continuous $200-\mu \mathrm{m}$ diameter gas nozzle, using typical backing pressures of 0.5 bar. The light emerging from the generated gas is propagated through a $200 \mathrm{~nm}$ aluminum filter in order to remove the remaining IR field, and then, the XUV is refocused by an $\mathrm{f}=750 \mathrm{~mm}$ toroidal mirror into the MBES using a $2 \mathrm{f}-2 \mathrm{f}$ configuration.

The remaining MIR light is recombined with the XUV on a hole mirror and then spatiotemporally overlapped, with variable delay, in a neon gas jet in the MBES for photoelectron time-of-flight spectroscopy. With this apparatus, we are able to record photoelectron spectral intensities that are proportional to the XUV spectral intensities, and using the reconstruction of attosecond beating by interference of two-photon transitions (RABBITT) method, we are able to measure the XUV GD. ${ }^{40}$ After retrieving the GD, the delays from the aluminum filter, neon detection gas, and HHG attochirp are removed. This retrieval process, which is discussed in more detail by Scarborough et al., ${ }^{15}$ works well in the energy range of $28-60 \mathrm{eV}$. Below $\approx 28 \mathrm{eV}$, the neon detection gas atomic delay calculation introduces systematic artifacts, and above $60 \mathrm{eV}$, the signal-to-noise ratio is too low to retrieve accurate GD results.

RABBITT provides a discrete sampling of the XUV GD with data points separated by $2 \hbar \omega$ in energy, where $\omega$ is the frequency of the IR driving field; for wavelengths near $1300 \mathrm{~nm}$, the sampling is approximately every $2 \mathrm{eV}$. This means that features that are on the order of or smaller than this become difficult to accurately characterize. To this end, we have developed a wavelengthscanning technique in which we tune the HHG driving wavelength (and thus the harmonic comb) and record RABBITT traces for each wavelength. We then combine the different measurements to produce a finely sampled measurement of the XUV GD. A more indepth explanation of the method and its applicability is provided in Appendix A.

For molecular-frame measurements, the alignment, or "kick" pulse, was propagated coparallel with the HHG driver and focused into the HHG gas source. The HHG pulse was then delayed relative to the kick pulse so that it was temporally overlapped with the half-revival of each molecule. For fine-tuning, a half waveplate was inserted into the kick beam at a fixed temporal delay to control the polarization of the kick relative to the HHG beam for angleresolved amplitude and GD measurements. We are unable to comment quantitatively on the degree of molecular alignment, as the measurement through HHG is not strictly linearly proportional to the degree of alignment. For our purposes, the shape of the distribution is narrow enough to discern significant changes in the amplitudes and group delays with $22.5^{\circ}$ steps.

\section{B. Theoretical methods}

We calculate the angle-dependent harmonic spectral intensity and phase for $\mathrm{CO}_{2}$ and OCS molecules using TDDFT as implemented in the software package Octopus. ${ }^{41}$ As described in more detail below, the driving laser field is linearly polarized and the molecular axis is oriented at angle $\theta$ relative to the laser polarization. We integrate the Kohn-Sham equations on a spatial grid and apply the local density approximation with average density selfinteraction correction (LDA-ADSIC) for the exchange-correlation potential. ${ }^{42}$ The calculations are converged using a time step of 0.05 a.u. and a grid spacing of 0.4 a.u., and the grid spans a rectangular box with dimensions of $390 \times 60 \times 60$ a.u. centered on the molecule and with the longest dimension along the laser polarization direction. In our simulations, we find $I p=14.55 \mathrm{eV}$ for $\mathrm{CO}_{2}$ and $I p=11.67 \mathrm{eV}$ for OCS (experimental values are $13.77 \mathrm{eV}$ and $11.17 \mathrm{eV}$, respectively ${ }^{43}$ ).

A computational challenge for all single-molecule calculations is the inherent interference between the contribution of multiple quantum paths in the HHG process. ${ }^{44}$ In experiments, macroscopic effects naturally select short trajectories. ${ }^{45-47}$ In the calculations, this interference is particularly detrimental when trying to extract targetspecific information from the spectral intensity and phase. To this end, we use an ionization seed in combination with the intense MIR field, in the form of an attosecond pulse train (APT) synthesized from odd harmonics of the MIR laser (harmonics 9-17 for $\mathrm{CO}_{2}$ and 7-15 for OCS). The APT dominates the ionization step in the HHG process and its subcycle timing is such that it strongly enhances the short quantum path contribution, thereby suppressing effects of multipath interferences. The calculations shown in this paper are all performed with a $6 \times 10^{13} \mathrm{~W} / \mathrm{cm}^{2}, 1500 \mathrm{~nm}$ MIR field, and APT intensities of $1.2 \times 10^{12} \mathrm{~W} / \mathrm{cm}^{2}$ for $\mathrm{CO}_{2}$ and $6 \times 10^{11} \mathrm{~W} / \mathrm{cm}^{2}$ for OCS. The MIR and APT intensities and wavelengths are chosen such that, although the APT dominates the ionization step, it has as small of an effect as possible on the subsequent electron dynamics, and the calculations span a similar range of harmonics energies to experimental results. The pulse intensity is ramped up with a $\sin ^{2}$ shape over 2 laser cycles. The carrier phase is such that the instantaneous field is zero at the end of the ramp-up.

We calculated the harmonic spectral intensity and phase from the Fourier transform $\mathcal{F}$ of the time-dependent acceleration signal $\mathbf{a}(t)$, after applying a window function $W(t)$. The window function helps us to further clean up the signal and suppress contributions from quantum paths longer than one cycle. $W(t)$ has a $\cos ^{4}(t)$ shape and selects the emission from the first half-cycle after the laser reaches its maximum intensity. For the spectral intensity, we include components oscillating both parallel and perpendicular to the laser 
polarization and thus show

$$
\left|\mathcal{F}\left[a_{\|} W\right](v ; \theta)\right|^{2}+\left|\mathcal{F}\left[a_{\perp} W\right](v ; \theta)\right|^{2},
$$

where $v$ is the harmonic frequency. To calculate HHG spectra from aligned rather than oriented OCS, we average the dipole-acceleration signal over angles $\theta$ and $\theta+\pi$. This is not necessary for the signal from the symmetric $\mathrm{CO}_{2}$ molecule.

To extract the target-specific spectral GD, similar to what is done in quantitative rescattering theory, we factorize the harmonic signal in the frequency domain ${ }^{12,37,48}$

$$
H H G(v ; \theta)=\sqrt{\frac{\Gamma(\theta)}{\Gamma_{r e f}}} \times H H G_{r e f}(v) \times \sigma(v ; \theta) \mathrm{e}^{i \phi(v ; \theta)},
$$

where $\Gamma$ is the energy-independent ionization yield, the " $r e f$ " subscripts label a generic "reference" system, and $\sigma$ and $\phi$ are the target-specific scattering cross section amplitude and phase, respectively. From Eq. (3), we extract the target-specific phase and GD, respectively, as

$$
\phi(v ; \theta)=\arg \left(\frac{H H G(v ; \theta)}{H H G_{r e f}(v)}\right) \text { and } G D=-\frac{\partial \phi}{\partial v} .
$$

For all TDDFT spectral analyses reported in this paper, our reference consists of a single-active-electron time-dependent Schrödinger equation calculation for a one-dimensional atom with a matching Ip interacting with an identical laser field, including the APT seed. Similar results were obtained using a matching two-dimensional reference. The reference calculations thus include both (i) generic features associated with the long-range tail of the Coulomb potential and (ii) systematic features associated with the seed. In the total GD, contributions from the parallel and perpendicular contributions are weighted by their spectral intensity.

\section{RESULTS AND DISCUSSION}

\section{A. Molecular-frame measurements-Spectral intensity}

We start by considering the angle dependence of the TCI minima in the spectral intensity, as shown for all three molecules in Fig. 2. Each spectrogram shows the results of a delay scan between the HHG and kick pulses across the half revival, allowing us to record the HHG spectrum as the molecular angle evolves from $-90^{\circ}$ to $0^{\circ}$ to $+90^{\circ}$. Asymmetries between positive and negative angles are attributed to the evolution of rotational wave packets at different probe delays. The delay scans have been smoothed with a SavitzkyGolay filter along the delay dimension to remove step-to-step noise, and the aluminum filter transmission and neon detection gas cross section have been removed. For $\mathrm{CO}_{2}$ and $\mathrm{N}_{2} \mathrm{O}$, we observe similar TCI features, namely, that the interference minimum reaches its lowest energies at $0^{\circ}$ and moves to higher energies as $\theta$ is increased. This is qualitatively consistent with the geometric expectation from Eq. (1), which predicts an evolution following $I_{p}+\alpha / \cos ^{2} \theta$, where $\alpha$ is a constant of proportionality. Remember that Eq. (1) explicitly states that $k_{e} \propto 1 / \cos \theta$, whereas the results in Fig. 2 are plotted as a function of emitted photon energy, which is proportional to $k_{e}^{2}$; hence, the experimental results are expected to be proportional to $1 / \cos ^{2} \theta$. The $0^{\circ}$ location of the minimum is slightly higher in $\mathrm{N}_{2} \mathrm{O}$ than in $\mathrm{CO}_{2}$. This is consistent with a smaller effective lobe separation in $\mathrm{N}_{2} \mathrm{O}$ for which the lobes are centered on the oxygen and the $\mathrm{N}-\mathrm{N}$ bond, whereas in $\mathrm{CO}_{2}$, they are centered on the two oxygen ends. A similar geometric TCI behavior is also present for OCS in Fig. 2(c), with a weak minimum starting at $35 \mathrm{eV}$ at $0^{\circ}$ and moving to higher energies as $\theta$ increases [see also Fig. 3(c)]. However, this behavior is much harder to recognize in OCS since the spectrum is dominated by a deep minimum near $43 \mathrm{eV}$ for angles up to about $45^{\circ}$.

The different angle dependence of the spectral yields for the three molecules is explored further in Figs. 3(a)-3(c). These spectra were recorded at a fixed delay corresponding to $0^{\circ}$ while rotating the polarization of the kick pulse to different values of $\theta$. This is a different method of rotating the molecules compared to that used in Fig. 2, which acts as a point of comparison and allows us to identify systematic errors in either of the spectral intensity measurement methods. All yields have been normalized by the near-featureless $90^{\circ}$ values. Figure 3 shows, in agreement with the observations in Fig. 2, that $\mathrm{CO}_{2}$ (a) and $\mathrm{N}_{2} \mathrm{O}$ (b) experience their deepest minima at $0^{\circ}$, with the minimum moving to higher energies for larger angles. Conversely, the minimum in OCS is largely constrained to the $30-50 \mathrm{eV}$ region, with a weak double minimum for angle $0^{\circ}$ at $35 \mathrm{eV}$ and $43 \mathrm{eV}$, and the deepest minimum found for angles $22.5^{\circ}$ and
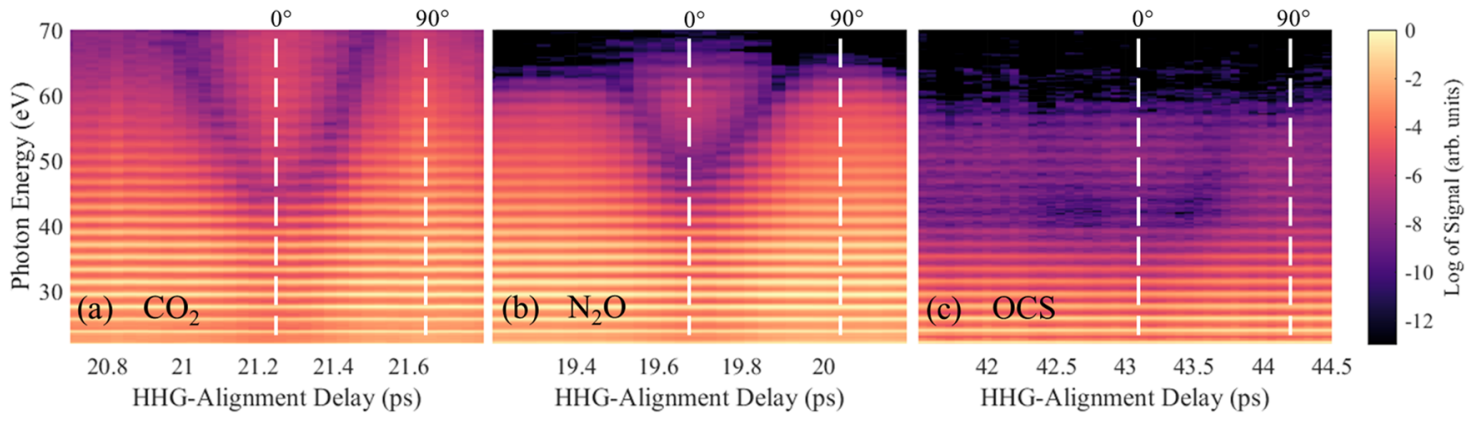

FIG. 2. Delay scans around the half revivals taken at $1300 \mathrm{~nm}$. Photoelectron spectra were recorded as a function of time delay between the alignment and high harmonic generation pulses, shown here after removal of the aluminum transmission and neon photoionization cross sections. The molecules can be thought of as smoothly evolving from $0^{\circ}$ at shorter delays to $90^{\circ}$ at longer delays, both of which are approximately marked with vertical, dashed white lines and labeled on the top axis. 


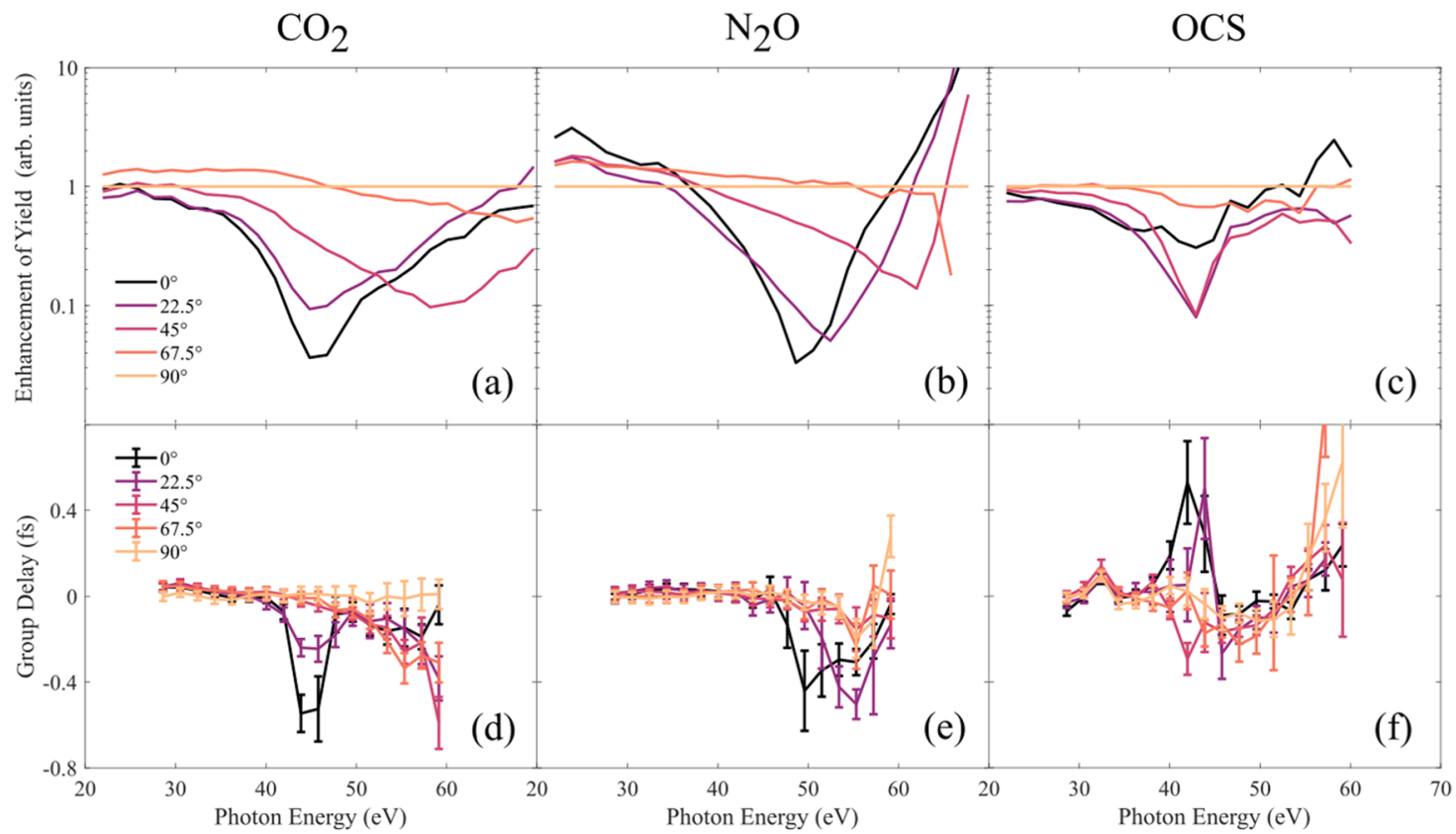

FIG. 3. [(a)-(c)] Enhancement of spectral yield relative to $90^{\circ}$ signal. [(d)-(f)] Group delay for different alignment angles for $\mathrm{CO}_{2}, \mathrm{~N}_{2} \mathrm{O}$, and OCS, respectively. Amplitude and group delay contributions from the aluminum filter, the neon detection gas, and the attochirp have been removed. See text for further discussion. Group delay data above $60 \mathrm{eV}$ were omitted due to low count rates resulting in poor statistics.

$45^{\circ}$, at $43 \mathrm{eV}$. Our results for $\mathrm{CO}_{2}$ and $\mathrm{N}_{2} \mathrm{O}$ are in agreement with those of earlier studies. ${ }^{16,17}$ In addition, in Appendix B, we show that the $0^{\circ}$ minimum positions are robust against changes in the laser intensity for all three molecules. All of the above support our interpretation that the interferences measured here are structural in nature and furthermore that the measured angular-variation of the TCI minimum in $\mathrm{CO}_{2}$ and $\mathrm{N}_{2} \mathrm{O}$ is primarily due to the geometric $k_{e} R \cos (\theta)$ term of Eq. (1). Furthermore, we do not find significant effects of the permanent dipole moment in $\mathrm{N}_{2} \mathrm{O}$, which, in principle, would give rise to intensity-dependent shifts via the Stark effect.

We interpret the different behavior of the OCS angular dependence compared to $\mathrm{CO}_{2}$ and $\mathrm{N}_{2} \mathrm{O}$ in the context of the chemical structure of the three molecular HOMOs. As shown in Fig. 1, the $\mathrm{HOMO}$ of $\mathrm{CO}_{2}$ is, by construction, symmetric, and $\mathrm{N}_{2} \mathrm{O}$ is nearly so, with little difference in electronic structure from swapping $\mathrm{C}-\mathrm{O}$ to $\mathrm{N}-\mathrm{N}$; both HOMOs are dominated by atomic $2 \mathrm{p}$ character in the LCAO basis. ${ }^{49}$ OCS, however, is much more asymmetric, with the sulphur atom contributing a $3 p$ orbital character to the HOMO. Following the qualitative framework of Eq. (1), the imbalance of the HOMO would be accounted for in the $\Delta \Phi$ term. The most prominent structure which imprints itself on the OCS HOMO comes from the sulphur $3 \mathrm{p}$ orbital. The $\mathrm{C}-\mathrm{S}$ bond can be thought to electronically fill the shell, making it isoelectronic with argon, which has a minimum in the photoionization cross section at a specific photon energy caused by a sign change in the RDME characterizing the transition between the ground state and a particular angular momentum channel; this manifests as a minimum in the total outgoing radial wavefunction. ${ }^{50,51}$ Using photoelectron spectroscopy, this minimum has been seen to extend to molecules that have $3 p$ character in the HOMO, including OCS. ${ }^{21}$ Because the molecular axis breaks the rotational symmetry of a Cooper minimum, the minimum is attributed as a "Cooper-like" minimum in the molecular case. Carlson et al. ${ }^{21}$ also justifies the use of the LCAO basis through charge density analysis, which attributes $97 \%$ of the atomic contribution to the HOMO to the $2 \mathrm{p}$ shell of oxygen and $3 \mathrm{p}$ shell of sulfur. As we will argue in further detail below, we believe we are seeing the combined effects of a Cooper-like minimum, which is nearly angle independent in its location, and a geometric TCI minimum which moves upward in energy as the angle increases.

As a final note on the angle-dependent yields, we, in general, observe a slower evolution of the energy of the TCI minimum with angle than that predicted by Eq. (1), for all three molecules. Empirically, we find a better match with

$$
\operatorname{TCI}(\theta) \approx I_{p}+\frac{\alpha}{|\cos \theta|^{\beta}},
$$

with $\beta \approx 1$. This deviation from the prediction of Eq. (1) is generally consistent with previous findings that plane-wave approximation of $\mathrm{RDME}$ often give poor quantitative predictions.

\section{B. Molecular-frame measurements-Spectral group delay}

We next focus on the angle-dependent GD measurements shown in Figs. 3(d)-3(f). The angle is varied in the same way as for the yield measurements in panels [(a)-(c)], thus allowing us to track the TCI behavior simultaneously in both the amplitude and phase. 
As a first observation from this comparison, we find that the angledependent GD of all three molecules indeed exhibits a TCI feature which mimics that of their angle-dependent yields. $\mathrm{CO}_{2}$ and $\mathrm{N}_{2} \mathrm{O}$, for example, both exhibit a minimum in the GD at the location of the amplitude minimum for all angles at which the two features can be discerned. The measured minima in the GD for $\mathrm{CO}_{2}$ and $\mathrm{N}_{2} \mathrm{O}$ correspond to a negative shift of the spectral phase of $\sim 1.5\left(\mathrm{CO}_{2}\right)$ and $\sim 2$ radians $\left(\mathrm{N}_{2} \mathrm{O}\right)$ at $0^{\circ}$. Interestingly, even though $\mathrm{N}_{2} \mathrm{O}$ is not perfectly symmetric and has a permanent dipole moment, we do not observe any meaningful difference between its TCI behavior and that of $\mathrm{CO}_{2}$. However, the OCS behavior is again starkly different from that of $\mathrm{CO}_{2}$ and $\mathrm{N}_{2} \mathrm{O}$. While the location of the OCS GD feature also matches that of the TCI minimum in the sense that it is strongly localized between 40 and $45 \mathrm{eV}$, its angle dependence is very different from the other two molecules: the OCS GD exhibits a maximum at angles of $0^{\circ}$ and $22.5^{\circ}$, which changes to a (shallower) minimum at $45^{\circ}$ and $67.5^{\circ}$. The $0^{\circ}$ GD maximum of OCS corresponds to a positive phase jump of $\sim 3$ radians.

Because OCS deviates in such a drastic manner from the other two molecules at $0^{\circ}$, it is important to perform a careful characterization of the GD feature with wavelength scanning for finer energy sampling. Shown in Fig. 4 are three wavelengths scans from 1270 to $1330 \mathrm{~nm}$ in $10 \mathrm{~nm}$ steps at $0^{\circ}$ for all three molecules. The results for OCS have been shifted by +300 as for clarity. With the combined results of the wavelength scans, it is clear that OCS has a smoothly varying maximum in the $\mathrm{GD}$ at $0^{\circ}$, whereas $\mathrm{CO}_{2}$ and $\mathrm{N}_{2} \mathrm{O}$ both have minima.

All of the results shown in Figs. 2-4 suggest that the Cooperlike minimum in OCS strongly influences the amplitude and phase of the molecular RDME through its interplay with the geometric TCI feature. We propose that the OCS results can in fact be interpreted in terms of a coherent sum of two structural features: (i) A geometric TCI minimum in the amplitude, accompanied by a maximum in the GD, which moves upward in energy with angle, similar to the prediction in Eq. (5), and (ii) a Cooper-like minimum in the amplitude, accompanied by a minimum in the GD, which is nearly angle-independent. As the TCI minimum "moves through"

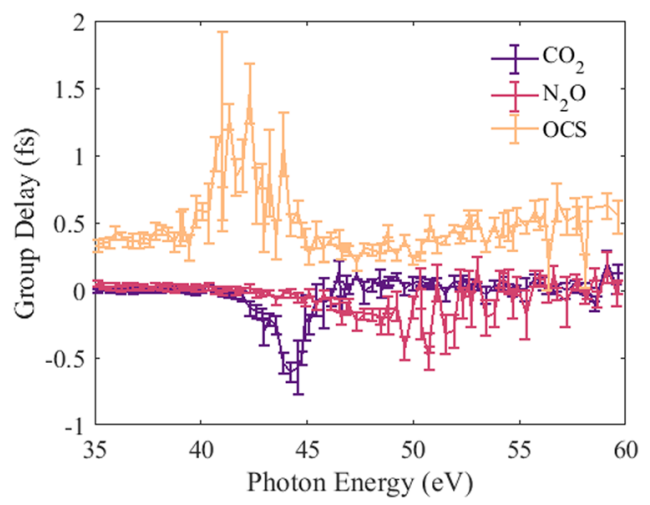

FIG. 4. Group delays at $0^{\circ}$ for all three molecules. The high harmonic driving wavelength was scanned from 1270 to $1330 \mathrm{~nm}$ for $0^{\circ} \mathrm{GD}$ measurements, and datasets were concatenated after attochirp removal. OCS has a +300 as shift added to it for clarity. the Cooper-like minimum, the two features can either add destructively or constructively, thereby increasing or decreasing the depth of the minimum. Similarly, the sum of the two features with opposite phase behavior can give rise to the observed sign change in the phase shift, from positive at small angles to negative at larger angles. We will discuss this interpretation in more detail in Sec. III E, where we present a conceptual model for the observed OCS behavior.

Finally, we note that prior to this paper, the only other molecular-frame GD measurements of TCI were performed in $\mathrm{CO}_{2}$ by Boutu et al. ${ }^{2}$ In that study, they measured the sign of the twocenter phase shift to be positive at $0^{\circ}$, in contrast to our measurements shown above. However, other measurements ${ }^{4,17}$ have indicated that for NIR wavelengths the interference effect observed by Boutu et al. is due to multiple orbital contributions to the HHG spectrum and as such cannot be directly compared to our measurement. A more comprehensive study comparing GD measurements in $\mathrm{CO}_{2}$ between NIR and MIR driving wavelengths will be the subject of a future paper.

\section{Unaligned measurements}

Because we ascribe the deviation of OCS from the patterns of $\mathrm{CO}_{2}$ and $\mathrm{N}_{2} \mathrm{O}$ to the overlapping of geometric TCI with the Cooperlike structure, it is instructive to confirm that the Cooper-like minimum survives in the unaligned molecular sample. In contrast, a purely geometric TCI minimum is expected to average out in both the amplitude and the phase when measuring harmonics from an unaligned sample. Figure 5(a) shows the spectral yields from the three unaligned samples, generated at $1300 \mathrm{~nm}$. Each spectrum is normalized to unity after correcting for the aluminum filter transmission and the neon detector photoionization cross section. The spectra for $\mathrm{CO}_{2}$ and $\mathrm{N}_{2} \mathrm{O}$ are relatively featureless until the cutoff of $\mathrm{N}_{2} \mathrm{O}$ is reached and until the aluminum filter $\mathrm{L}_{2,3}$ edge $(\approx 72 \mathrm{eV})$ is reached for $\mathrm{CO}_{2}$. On the other hand, OCS departs from the spectra of the other two around $30 \mathrm{eV}$ and descends rapidly until a "kink" at $\approx 43 \mathrm{eV}$, indicated by a vertical dashed line. After this point, the spectrum exhibits a flat plateaulike structure until the cutoff is reached. We have found that the location of this feature in unaligned OCS is independent of the driving laser intensity at $1300 \mathrm{~nm}$ and the wavelength when measured at $1500 \mathrm{~nm}, 1700 \mathrm{~nm}$, and $2000 \mathrm{~nm}$ (not shown in the figure). Across the $40-50 \mathrm{eV}$ region, the unaligned OCS spectral yield decreases by approximately two orders of magnitude compared to that of the other two molecules. The suppression at high energies is in large part due to the Cooper-like minimum in the OCS RDME amplitude and is in qualitative agreement with the results of photoionization experiments performed in all three molecules.

Figure 5(b) shows the combined results of GD wavelength scans for all three unaligned samples. Driving wavelengths in the range 1270-1330 nm with $10 \mathrm{~nm}$ steps were used. The OCS results demonstrate a broad minimum of $\approx-150$ as in GD around $43 \mathrm{eV}$, coincident with the spectral kink in the unaligned OCS spectral intensity. Over the same spectral region, the unaligned $\mathrm{CO}_{2}$ and $\mathrm{N}_{2} \mathrm{O}$ samples are featureless.

The fact that OCS retains a minimum in both the amplitude and the GD even in the unaligned sample is another indication that we are observing a relatively angle-independent Cooper-like 


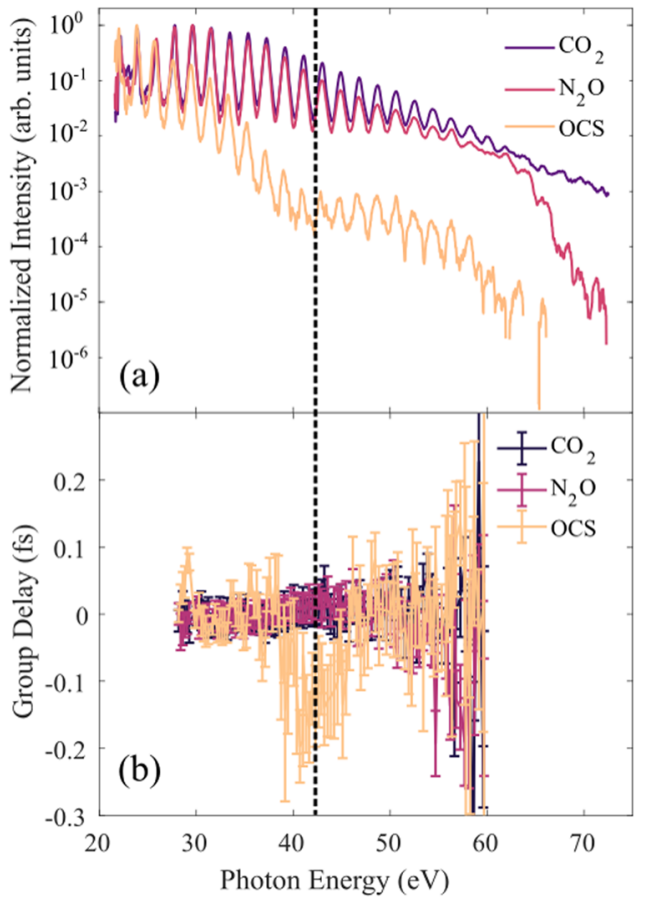

FIG. 5. Spectral intensities (a) and GD (B) for unaligned samples of $\mathrm{CO}_{2}, \mathrm{~N}_{2} \mathrm{O}$, and OCS. In (b), the GD is recorded every $10 \mathrm{~nm}$ using a driving wavelength of $1270-1330 \mathrm{~nm}$, and concatenated into a single dataset as described in the text.

minimum in the RDME. It is also interesting to note that the location and size of the OCS GD minimum is in agreement with previous measurements of $\mathrm{Ar}$ and $\mathrm{CH}_{3} \mathrm{Cl}$, which exhibit $3 \mathrm{p}$ valence character, where Cooper-like minimum positions were previously found to be between 40 and $50 \mathrm{eV}$ with GD minima between -100 and -200 as. $^{15,51}$

\section{TDDFT simulations}

We next consider the TDDFT-calculated results for the HHG spectral intensity and GD for $\mathrm{CO}_{2}$ and OCS, displayed in Fig. 6. Panels (a) and (b) show the harmonic spectral yields for $\mathrm{CO}_{2}$ and OCS, respectively. These calculated yields are comparable to the experimental angle-dependent yields shown in Figs. 2(a) and 2(c). Each angle-dependent spectrum has been divided by a smoothed, featureless, incoherent average over all calculated angles, which allows us to follow the TCI minimum at energies beyond the cutoff. For comparison, the dotted line labels the empirical prediction of Eq. (5), with values for $\alpha$ and $\beta$ given in the caption. Generally speaking, when there is a minimum, we observe a good qualitative agreement between TDDFT results and the empirical prediction. For $\mathrm{CO}_{2}$, this also means we have good agreement with the experimental results shown in Figs. 2(a) and 3(a). Comparing TDDFT results for $\mathrm{CO}_{2}$ and OCS, we also observe a lower energy TCI feature at $0^{\circ}$ in the latter, again in agreement with experiments, and consistent with a larger distance between the two centers in Eq. (1).

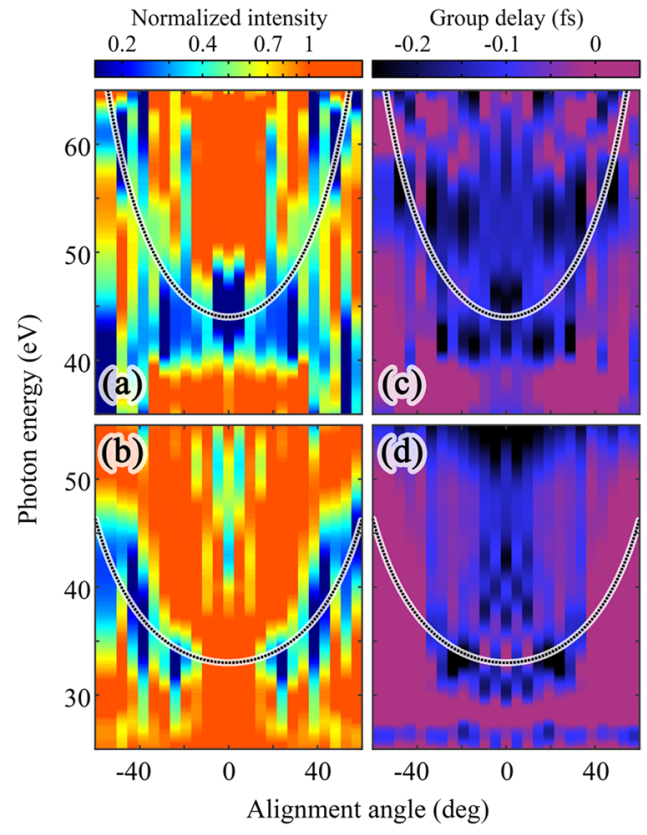

FIG. 6. Alignment-angle dependent spectral intensity [(a) and (b)] and group delay [(c) and (d)] obtained from TDDFT simulations in $\mathrm{CO}_{2}[(\mathrm{a})$ and (c)] and OCS [(b) and (d)]. In all panels, the dotted curve labels the empirical $\mathrm{TCl}$ minimum of Eq. (5) with $\alpha=44 \mathrm{eV}-\mathrm{Ip}$ and $\beta=1$ for $\mathrm{CO}_{2}$ and $\alpha=33 \mathrm{eV}-\mathrm{Ip}$ and $\beta=0.7$ for OCS. Spectral intensities are normalized with a smoothed (featureless) incoherent average over computed angles, to reveal the $\mathrm{TCl}$ minimum beyond cut-off energies.

However, in general, the OCS results exhibit qualitative and quantitative differences with the experiments. As we have discussed above, the experimental TCI minimum around $43 \mathrm{eV}$ remains visible at most angles and is most prominent around $30^{\circ}$. In the simulation, while we match the relative absence of a minimum below $20^{\circ}$, the minimum for larger angles behaves like a TCI minimum, although the energy increases somewhat slower with angle than for $\mathrm{CO}_{2}$. Second, and consistent with the first observation, the calculations do not exhibit clear signs of the Cooper-like minimum seen in the experimental results (the shallow minimum from 40 to $50 \mathrm{eV}$ at small angles cannot be conclusively assigned to a Cooper-like minimum). It is not entirely surprising that the TDDFT calculations do not reproduce the Cooper-like minimum, as especially the location of such a minimum is notoriously difficult to predict and requires a very accurate description of the continuum wave functions. ${ }^{31}$ The absence of this Cooper minimum in the calculated OCS response means that aligned OCS behaves somewhat generically like $\mathrm{CO}_{2}$ in terms of the location of the TCI minimum, except with a slightly larger effective center-to-center separation leading to the lower energy of the $0^{\circ}$ minimum. It is worth noting that for oriented OCS, we see a clear difference in the harmonic response from consecutive half-cycles as the continuum electron wave packet has been released from and scatters on opposite ends of the molecule. In particular, both the overall yield and the location of the TCI minimum are different from half-cycle to halfcycle. Since the current experiment only addresses aligned, but not 
oriented, OCS, we will leave discussions of oriented OCS for a future study. ${ }^{5}$

Finally, we discuss the calculated angle-dependent GDs as shown in Fig. 6(c) for $\mathrm{CO}_{2}$ and Fig. 6(d) for OCS. The solid curves show the same empirical geometric expectation as that in panels (a) and (b), respectively. For small alignment angles, comparison of panels [(a) and (b)] and [(c) and (d)] shows the GD also approximately follows the geometric expectation: the $\mathrm{CO}_{2}$ (OCS) GD exhibits a minimum near $44 \mathrm{eV}(33 \mathrm{eV})$ for angles up to about $30^{\circ}$. For larger angles, though, the GD does not exhibit clear minima that can be associated with structural features. In fact, we find that it is, in general, much more difficult to extract the GDs than the yields from the TDDFT computations and that the details of the APT and its timing have a greater influence on the extracted GD than the yields. This will be explored in more detail in future studies. ${ }^{56}$ We note that, again, since calculated OCS does not have a Cooper minimum, the two molecules behave quite similarly, both exhibiting an approximate -250 as decrease in the GD across the minimum. This is in contrast to the experimental results, where the $\mathrm{CO}_{2} \mathrm{GD}$ decreases and the OCS GD increases across the minimum at $0^{\circ}$.

\section{E. Conceptual model}

To further understand the experimental results, and, in particular, to study the interplay between the geometric TCI and a Cooper-like features in OCS, we build a conceptual model for the harmonic spectral amplitude and phase, guided by the experimental measurements and parameters. The overall results, compared to experimental measurements, are shown in Fig. 7 for $\mathrm{CO}_{2}$ and Fig. 8 for OCS. The conceptual model is built from the factorization in Eq. (3), where the reference is taken as a featureless spectral amplitude that has been fit to the $90^{\circ}$ experimental signal [see the insets in panel (a) of Figs. 7 and 8]. We include an angle-dependent ionization yield $\Gamma(\theta)$ that gives a slight preference to $90^{\circ}$ vs $0^{\circ}$, consistent with the measured relative spectral intensities for HHG energies below the TCI minimum. To match experimental conditions, we also include and average over an alignment distribution in angleresolved data. Further technical details about the model and choice of parameters are given in Appendix $\mathrm{C}$.

For $\mathrm{CO}_{2}$, the key elements of TCI are angle-dependent features in the amplitude " $\sigma$ " and phase " $\phi$ " of Eq. (3). We model them with Gaussian shapes that move geometrically as the molecule is rotated following the empirical formula of Eq. (5) (see Appendix C for details). Figure 7 shows that this model captures all the main elements of the experimental data we have discussed so far: (i) the TCI minimum barely moves in energy between $0^{\circ}$ and $22.5^{\circ}$, and (ii) the minimum and the GD feature both start out narrow and deep and become broader and shallower at larger angles. We use a geometric feature with constant depth and width in our model (see Appendix C) and this broadening is the result of the molecular-alignment distribution: the TCI gets more spread out at larger $\theta$, where the minimum moves faster with the alignment angle. The agreement between this simple model and the experimental results means that it is a good approximation to think of TCI as a generic and robust structural interference feature. Although the angle dependence of the location of the minimum is, in general, different from the simplest plane-wave-based geometric expectation of
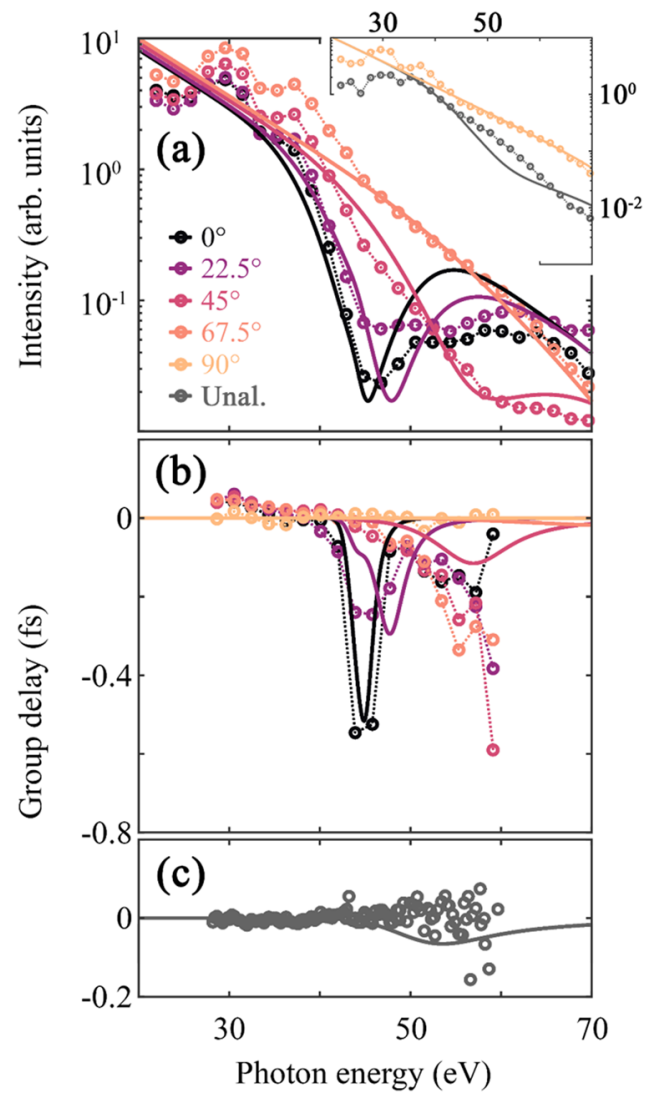

FIG. 7. Comparison between our conceptual model with a geometric $\mathrm{TCl}$ feature (solid lines-see Sec. III E) and experimental measurements (circles with dotted curves) for $\mathrm{CO}_{2}$. Spectral intensities are shown in panel (a) and target-specific group delays for aligned and unaligned samples are shown in panels (b) and (c), respectively.

Eq. (1), the effect of the TCI on the amplitude and phase is generic across many alignment angles.

The simple model also allows us to approximately calculate the yield and GD from the unaligned sample, as shown in Fig. 7 [panel (a) inset and panel (c)], respectively, along with the corresponding experimental results. On smaller scales, the apparent reminiscence of a structural feature above $50 \mathrm{eV}$ in the unaligned spectral intensity is a result of the qualitative conceptual model we use here and does not carry further information on the TCI influence on unaligned signals. Figure 7 [(a), inset] shows that compared to the reference, the unaligned yield is dampened above the TCI minimum, and (c) the GD in the unaligned signal is almost completely washed out. The dampened yield at high energies is the only remaining trace of TCI in unaligned targets. It can be understood as a macroscopic or ensemble effect, where the HHG contributions from molecules at different alignment angles are out of phase over a large range of energies because of the phase shift which moves in energy. This means that for energies above the $0^{\circ}$ minimum, there will be a number of molecules which has undergone the phase shift and a number which has not. We note that while, in principle, we also could calculate an "unaligned" signal using the coherent average over the 


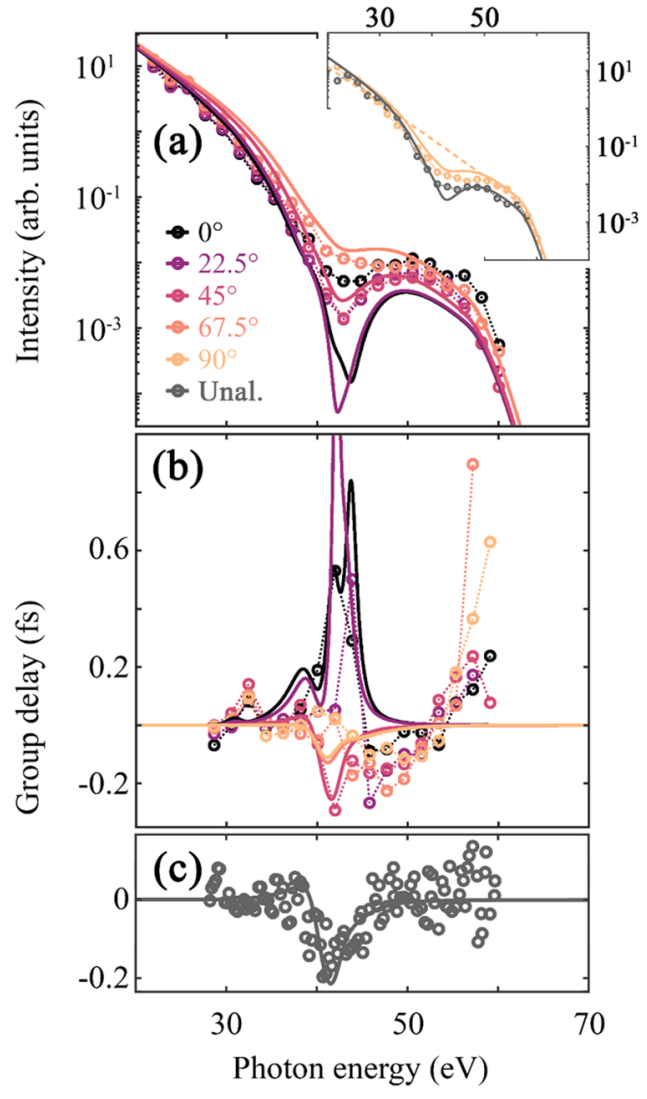

FIG. 8. Similar comparison between the conceptual model and experiments for OCS as in Fig. 7 (solid curves/circles for theory/experiments). Here, the geometric $\mathrm{TCl}$ feature is augmented with an angle-independent Cooper-like contribution; in the inset of (a), the $90^{\circ}$ spectra are shown with and without the Cooper-like feature (solid and dashed curves, respectively).

TDDFT calculations at different angles, in practice, the TDDFT results have too much variability from angle to angle to get a meaningful average. This can be seen from Fig. 6, for which the geometric expectation is only followed on average; for individual angles, the calculated minimum does not, in general, overlap exactly with the expectation.

We build on the success of the conceptual model for $\mathrm{CO}_{2}$ to consider the more complex case of OCS. For OCS, we include both a similar angle-dependent TCI feature as described above, in this case with a positive phase shift, and an angle-independent negative phase-shift feature associated with the Cooper-like contribution. The results are displayed in Fig. 8 (see Appendix $C$ for technical details). Here as well, the results reproduce key features of experimental measurements and help us shed additional light on them: (i) the features in the spectral intensity and GD barely move with the alignment angle and are most prominent when the two components align in energy. We attribute this to the TCI and Cooperlike contributions compensating each other when they are separated in energy. (ii) The GD rapidly changes sign with increasing alignment angle. This is fostered by interference between the TCI and Cooper-like contributions around energies where they have similar amplitude. (iii) In the unaligned signals, shown in panel (a) inset and panel (c), we now retain signatures of the Cooper-like minimum in both spectral intensity and phase; the inset shows the $90^{\circ}$ intensities both with (solid curve) and without (dashed curve) the Cooper-like contribution. The unaligned amplitude both exhibits the Cooper-like minimum itself and, similar to the case in $\mathrm{CO}_{2}$, is damped at high energies relative to the $90^{\circ}$ signal. In the GD, the angle-dependent geometric phase feature has been averaged out, leaving only the Cooper-like negative phase shift. The good agreement between this model and the experimental results, again, supports our interpretation of the OCS behavior as resulting from the interference between a generic TCI feature that moves through a nearly angle-independent Cooper-like feature as the alignment angle changes.

\section{SUMMARY}

We have investigated structural quantum interferences in $\mathrm{CO}_{2}$, $\mathrm{N}_{2} \mathrm{O}$, and OCS molecular samples through amplitude- and phaseresolved HHS. In all three molecules, we see evidence of a geometric TCI effect for which the interference minimum increases in energy with the alignment angle. In OCS we, in addition, observe a nearly angle-independent Cooper-like feature which interferes with the TCI feature in different ways at different angles. Our results validate the qualitative picture of Eq. (1) which provides a framework for the symmetric and near-symmetric cases of $\mathrm{CO}_{2}$ and $\mathrm{N}_{2} \mathrm{O}$, although we find that the TCI location increases more slowly with angle than predicted by Eq. (1). Our measurements in $\mathrm{N}_{2} \mathrm{O}$, along with the intensity independence of the minima, confirm that the $\Delta \Phi\left(k_{e}, \theta\right)$ term is not strongly affected by the laser field, suggesting that the difference between $\mathrm{CO}_{2}$ and OCS is dominantly structural. Investigation of the high-harmonic spectral intensities shows a strong suppression in OCS in both unaligned spectra and all angles of molecular-frame spectra relative to the other molecules, consistent with the Cooper-like mechanism mentioned above. Measurements of the GD further show the uniqueness of OCS in this work. This is manifested in the sign change of the GD feature for different angles, as the TCI minimum moves through the Cooperlike minimum and changes the interference. The interpretation of the observed results as an interference between TCI and Cooperlike features is supported by our conceptual model, which used generic spectral behaviors for both features and led to good qualitative and semiquantitative agreement with the experimental results. Finally, we presented TDDFT calculations in good qualitative agreement with the $\mathrm{CO}_{2}$ measurements, including reproducing the negative GD feature. In OCS, the TDDFT calculations also predict an angle-dependent TCI feature which can be recognized in the experimental results; however, we do not recover the Cooper-like feature which means that we found less overall agreement with the experimental results. Overall, our finding here that TDDFT calculations recover structural features such as the TCI minimum, combined with recent work showing that ultrafast charge migration can also be well represented by TDDFT, ${ }^{38,39}$ suggests that TDDFT calculations may be able to explore charge migration through HHS.

These results emphasize the importance of multidimensional measurements when investigating structural, and by extension dynamical, behavior: only through measurements of both spectral 
intensity and GD are the TCI and Cooper-like mechanisms fully characterized in OCS, and even then, only by comparison to other molecules are these characterizations validated. As future studies of molecular HHS examine larger, more complex molecules, such thorough characterizations may become increasingly important to elucidate the mechanisms involved.

\section{ACKNOWLEDGMENTS}

This work was supported by the U.S. Department of Energy, Office of Science, Office of Basic Energy Sciences, under Award No. DE-SC0012462. D.K. was supported by the U.S. Department of Energy, Office of Science, Basic Energy Sciences, under Award No. DE-FG02-04ER15614. High-performance computational resources were provided by the Louisiana Optical Network Initiative and the High Performance Computing center at Louisiana State University. L.F.D. acknowledges support from the Edward and Sylvia Hagenlocker Chair. The authors thank Robert R. Jones for fruitful discussions.

\section{APPENDIX A: WAVELENGTH SCANNING}

A critical parameter for wavelength tuning in resonance spectroscopy is the harmonic separation $2 \omega_{0}$ relative to the energetic width $\Delta E$. This complication arises due to the fact that, in RAB$\mathrm{BITT}$, only changes in phase are directly measured. In order to interpret this physically, the discrete derivative approximation is used to measure GD,

$$
G D=-\frac{\partial \phi}{\partial v} \cong-\frac{\Delta \phi}{2 \hbar \omega_{0}} .
$$

If $2 \hbar \omega_{0} \gg \Delta E$ such that only a single harmonic can be incident on the resonance in each RABBITT scan, then the discrete derivative is a poor approximation, and the phase (not the GD) is more directly probed. In the other extreme where $2 \hbar \omega_{0} \ll \Delta E_{R}$, multiple harmonics can sample the resonance at once and the discrete derivative approximation to the GD is accurate, meaning that the shape of the reconstructed GD will reflect the shape of the GD of the resonant structure.

In this work, we operate on the border of these regimes. In Fig. 3(a), we sample the TCI of $\mathrm{CO}_{2}$ with only 2-3 points, making the interpretation unclear; in Fig. 3(b), the structure in $\mathrm{N}_{2} \mathrm{O}$ is broader, and more representative of GD. For the purposes of precisely, quantitatively probing GD, our color scans in this regard may require additional convolution. However, toward the goal of providing finer sampling to confirm a smoothly varying positive or negative feature in the GD, the scans serve the intended purpose.

\section{APPENDIX B: INTENSITY DEPENDENCE OF $0^{\circ}$ MINIMA}

Figure 9 shows the spectral yield at $0^{\circ}$ at different driving laser intensities. Because the absolute spectral intensities vary considerably with changing intensity, all the spectra shown in Fig. 9 have been normalized to the unaligned intensities such that their enhancements relative to unaligned caused by the TCI is presented. These plots show that there is no obvious intensity-dependence to
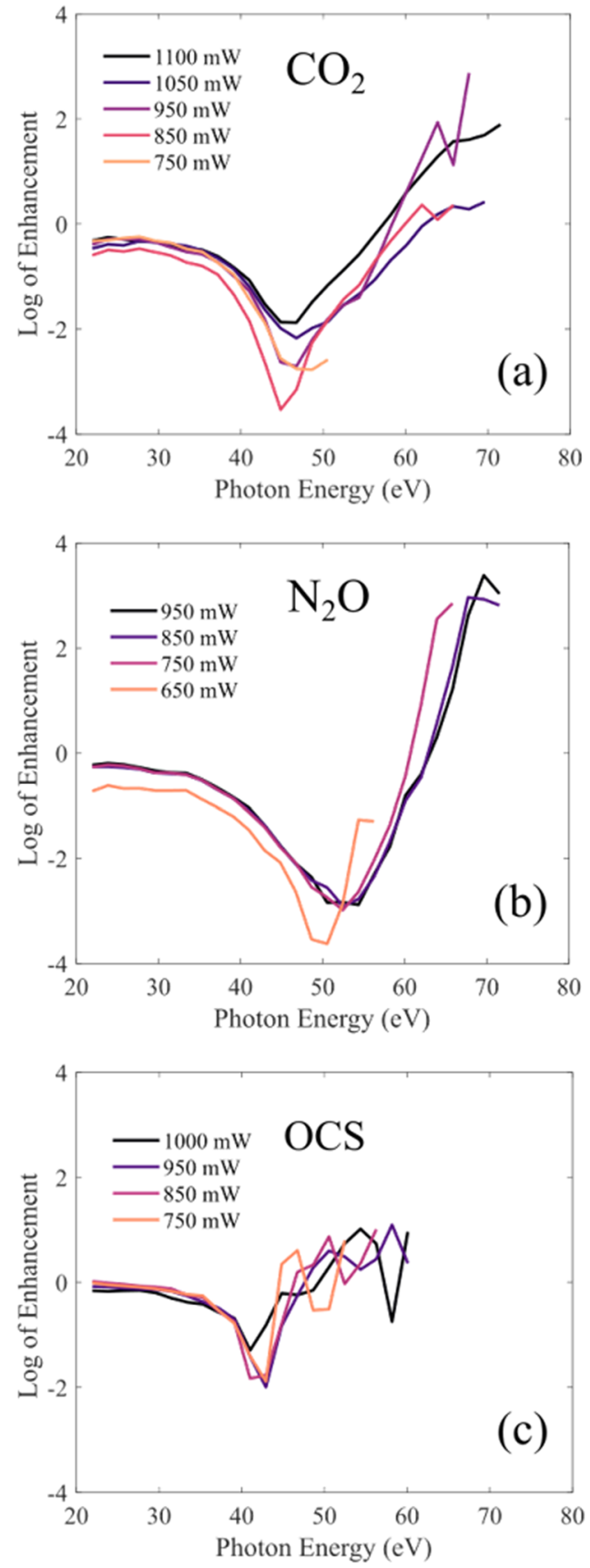

FIG. 9. Intensity independence of the interference position. Enhancement curves at $0^{\circ}$ relative to unaligned signals for all three molecules are essentially unchanged as the laser intensity is varied.

the minima positions. At the lowest intensity for $\mathrm{N}_{2} \mathrm{O}$, a slight shift is observed but this is more than likely due to the normalization procedure. As discussed above, the $0^{\circ}$ spectrum experiences a cutoff extension relative to unaligned, which means that the cutoff region at $0^{\circ}$ does not have a high-statistics region to normalize against. So as 
the cutoff approaches the interference, normalizing to the unaligned spectrum obscures the resonant feature. This problem is not seen in the other two molecules because their interferences are farther from the cutoff. All of the above results for $\mathrm{CO}_{2}$ and $\mathrm{N}_{2} \mathrm{O}$ are in agreement with those of earlier studies; ${ }^{16,17}$ therefore, it is reasonable to conclude that the measured angular variation of the interferences in $\mathrm{CO}_{2}$ and $\mathrm{N}_{2} \mathrm{O}$ is primarily due to the $k_{e} R \cos (\theta)$ term of Eq. (1). In contrast, the localized nature of the OCS interference enhancement indicates a structural interference in OCS that has additional angular variation from the phase difference $\Delta \Phi$ : not inconsistent with a Cooper-like minimum.

\section{APPENDIX C: CONCEPTUAL MODEL}

Looking back at Eq. (3), from the reference signal $H_{H} G_{r e f}(v)$, we see that the angle-resolved HHG spectral amplitude and GD are completely determined by the ionization yield $\Gamma(\theta)$, and the targetspecific amplitude $\sigma(v ; \theta)$ and phase $\phi(v ; \theta)$. Similar to Fig. 3, we calibrate the reference against the $90^{\circ}$ signal. More specifically, we define $H H G_{r e f}$ as a featureless-both in amplitude and phase-fit against $90^{\circ}$ experimental results (see the insets of Fig. 7) and normalize it by its yield $\Gamma\left(90^{\circ}\right)$. For the angle-dependent ionization yield, we use a trigonometric expansion

$$
\Gamma(\theta)=a_{0}+a_{1} \sin ^{2} \theta,
$$

where the coefficients $a_{0}$ and $a_{1}$ were selected to match relative spectral intensities at $0^{\circ}$ and $90^{\circ}$ below the TCI. For $\mathrm{CO}_{2}$ (OCS), we use a ratio $a_{0}^{2}: a_{1}^{2}=1: 0.3(1: 0.2)$. The angle dependence of the ionization yields plays a minor role in our results and is kept mostly for consistency with the full factorization of Eq. (3) and for adaptability to other compounds with a strong ionization angle dependence.

For $\mathrm{CO}_{2}$, TCI is modeled as a generic feature in $\sigma$ and $\phi$ that moves geometrically as the molecule is rotated following the empirical equation (5) with $\alpha=44 \mathrm{eV}-I p$ and $\beta=1$, and consistent with experimental observations (see discussions in Secs. III A and III B). More specifically, for the spectral amplitude, we choose

$$
\sigma(v ; \theta)=1-\left(1-\sigma_{\text {min }}\right) \exp \left(-\frac{(v-T C I(\theta))^{2}}{2 \sigma_{a}^{2}}\right),
$$

where the depth $\sigma_{\min }=\sqrt{4 \times 10^{-2}}$ and width $\sigma_{a}=5 \mathrm{eV}$. For the spectral GD, we choose

$$
G D(v ; \theta)=\tilde{\phi}_{t o t} \exp \left(-\frac{(v-T C I(\theta))^{2}}{2 \sigma_{p}^{2}}\right),
$$

and the phase is recovered by integration over harmonic energies. Here $\tilde{\phi}_{t o t}$ is set to obtain a total phase variation of $-0.7 \times \pi$ radians and $\sigma_{p}=0.9 \mathrm{eV}$. The total phase variation is determined from the damping in the unaligned signal above the TCI [see the inset of Fig. 7(a)], while all other parameters are determined from the measurements at $0^{\circ}$. Finally, to account for imperfect alignment in experimental measurements, results are averaged over a $\cos ^{2}$ distribution with a $40^{\circ}$ FWHM spread.

For OCS, in addition to the geometric TCI, an angleindependent electronic-structure feature is coherently added to account for the Cooper-like component. The relative weight between the two components is determined from the HOMO asymmetry between the two ends of the molecules, which we set to 0.35:1 (TCI:Cooper-like) ratio. Because they overlap, and interfere, in energy, it is hard to precisely calibrate each component independently. Instead, we choose to use the same parameter for OCS TCI as in $\mathrm{CO}_{2}$, with only the total phase variation sign $(+0.7 \times \pi \mathrm{rad})$ and minimum energy at $0^{\circ}(\alpha=31 \mathrm{eV}-I p)$ adjusted to reflect the experimental data. For the Cooper-like component, we use similar generic features in amplitude and GD fixed at $41 \mathrm{eV}$ with $\sigma_{\min }=\sqrt{5 \times 10^{-2}}, \sigma_{a}=5 \mathrm{eV}$, and $\tilde{\phi}_{\text {tot }}=-0.35 \times \pi$ rad. These parameters were set to match experimental results in unaligned samples and the sharp variations in the GD of aligned samples. Finally, like in $\mathrm{CO}_{2}$, a $\cos ^{2} 40^{\circ}$-FWHM distribution is used to describe the alignment distribution.

\section{REFERENCES}

${ }^{1}$ X. Zhou, R. Lock, W. Li, N. Wagner, M. M. Murnane, and H. C. Kapteyn, Phys, Rev. Lett. 100, 073902 (2008).

${ }^{2}$ W. Boutu, S. Haessler, H. Merdji, P. Breger, G. Waters, M. Stankiewicz, L. J. Frasinski, R. Taieb, J. Caillat, A. Maquet, P. Monchicourt, B. Carre, and P. Salieres, Nat. Phys. 4, 545 (2008).

${ }^{3}$ O. Smirnova, Y. Mairesse, S. Patchkovskii, N. Dudovich, D. Villeneuve, P. Corkum, and M. Ivanov, Nature 460, 972 (2009).

${ }^{4}$ H. J. Wörner, J. B. Bertrand, P. Hockett, P. B. Corkum, and D. M. Villeneuve, Phys. Rev. Lett. 104, 233904 (2010).

${ }^{5}$ O. Smirnova and M. Ivanov, Attosecond and XUV Spectroscopy: Ultrafast Dynamics and Spectroscopy, edited by T. Schultz and M. Vrakking (Wiley, 2014), Chap. 7.8, ISBN: 978-3-527-41124-5.

${ }^{6}$ P. M. Kraus, B. Mignolet, D. Baykusheva, A. Rupenyan, L. Horný, E. F. Penka, G. Grassi, O. I. Tolstikhin, J. Schneider, F. Jensen, L. B. Madsen, A. D. Bandrauk, F. Remacle, and H. J. Wörner, Science 350, 790 (2015).

${ }^{7}$ X. F. Li, A. L'Huillier, M. Ferray, L. A. Lompré, and G. Mainfray, Phys. Rev. A 39, 5751 (1989).

${ }^{8}$ A. L'Huillier and P. Balcou, Phys. Rev. Lett. 70, 774 (1993).

${ }^{9}$ K. J. Schafer, B. Yang, L. F. DiMauro, and K. C. Kulander, Phys. Rev. Lett. 70, 1599 (1993).

${ }^{10}$ P. B. Corkum, Phys. Rev. Lett. 71, 1994 (1993).

${ }^{11}$ J. Itatani, J. Levesque, D. Zeidler, H. Niikura, H. Pepin, J. Keiffer, P. Corkum, and D. M. Villeneuve, Nature 432, 867 (2004).

${ }^{12}$ C. Lin, A.-T. Le, Z. Chen, T. Morishita, and R. Lucchese, J. Phys. B: At., Mol. Opt. Phys. 43, 122001 (2010).

${ }^{13}$ S. Haessler, J. Caillat, W. Boutu, C. Giovanetti-Teixeira, T. Ruchon, T. Auguste, Z. Diveki, P. Breger, A. Maquet, B. Carré, R. Taeb, and P. Salières, Nat. Phys. 6, 200 (2010).

${ }^{14}$ S. B. Schoun, A. Camper, P. Salières, R. R. Lucchese, P. Agostini, and L. F. DiMauro, Phys. Rev. Lett. 118, 033201 (2017).

${ }^{15}$ T. Scarborough, T. Gorman, F. Mauger, P. Sándor, S. Khatri, M. Gaarde, K. Schafer, P. Agostini, and L. DiMauro, Appl. Sci. 8, 1129 (2018).

${ }^{16}$ C. Vozzi, M. Negro, F. Calegari, G. Sansone, M. Nisoli, S. De Silvestri, and S. Stagira, Nat. Phys. 7, 822 (2011).

${ }^{17}$ A. Rupenyan, P. M. Kraus, J. Schneider, and H. J. Wörner, Phys. Rev. A 87, 031401 (2013).

${ }^{18}$ M. Labeye, F. Risoud, C. Lévêque, J. Callait, A. Maquet, T. Shaaran, P. Salières, and R. Taïeb, Phys. Rev. A 99, 013412 (2019).

${ }^{19}$ A. Etches, M. B. Gaarde, and L. B. Madsen, Phys. Rev. A 84, 059903 (2011).

${ }^{20}$ M. D. Śpiewanowski and L. B. Madsen, Phys. Rev. A 89, 043407 (2014).

${ }^{21}$ T. A. Carlson, M. O. Krause, and F. A. Grimm, J. Chem. Phys. 77, 1701 (1982).

${ }^{22}$ T. A. Carlson, M. O. Krause, W. A. Svensson, P. Gerard, F. A. Grimm,

T. A. Whitley, and B. P. Pullen, Z. Phys. D: At., Mol. Clusters 2, 309 (1986).

${ }^{23}$ M. C. H. Wong, J.-P. Brichta, and V. R. Bhardwaj, Phys. Rev. A 81, 061402 (2010). 
${ }^{24}$ M. C. H. Wong, A.-T. Le, A. F. Alharbi, A. E. Boguslavskiy, R. R. Lucchese, J.-P. Brichta, C. D. Lin, and V. R. Bhardwaj, Phys. Rev. Lett. 110, 033006 (2013).

${ }^{25} \mathrm{M}$. G. White, K. T. Leung, and C. E. Brion, J. Electron Spectrosc. Relat. Phenom. 23, 127 (1981).

${ }^{26}$ P. M. Kraus, A. Rupenyan, and H. J. Wörner, Phys. Rev. Lett. 109, 233903 (2012).

${ }^{27}$ E. P. Fowe and A. D. Bandrauk, Phys. Rev. A 81, 023411 (2010).

${ }^{28}$ E. Penka Fowe and A. D. Bandrauk, Phys. Rev. A 84, 035402 (2011).

${ }^{29}$ X. Chu and G. C. Groenenboom, Phys. Rev. A 85, 053402 (2012).

${ }^{30}$ D. Dundas, J. Chem. Phys. 136, 194303 (2012).

${ }^{31}$ J. Mauritsson, M. B. Gaarde, and K. J. Schafer, Phys. Rev. A 72, 013401 (2005).

${ }^{32}$ M. Ruberti, P. Declevab, and V. Averbukha, Phys. Chem. Chem. Phys. 20, 8311 (2018).

${ }^{33}$ X. Li, D. J. Haxton, M. B. Gaarde, K. J. Schafer, and C. W. McCurdy, Phys. Rev. A 93, 023401 (2016)

${ }^{34}$ B. Augstein and C. Faria, J. Mod. Opt. 58, 1173 (2011).

${ }^{35}$ A.-T. Le, R. R. Lucchese, and C. D. Lin, Phys. Rev. A 87, 063406 (2013).

${ }^{36}$ B. Bruner, Z. Mašín, M. Negro, F. Morales, D. Brambila, M. Devetta, D. Faccialà, A. Harvey, M. Ivanov, Y. Mairesse, S. Patchkovskii, V. Serbinenko, H. Soiferf, S. Stagira, C. Vozzi, N. Dudovich, and O. Smirnova, Faraday Discuss. 194, 369 (2016).

${ }^{37}$ F. Mauger, P. M. Abanador, K. Lopata, K. J. Schafer, and M. B. Gaarde, Phys. Rev. A 93, 043815 (2016).

${ }^{38}$ A. Bruner, S. Hernandez, F. Mauger, P. Abanador, D. LaMaster, M. Gaarde, K. Schafer, and K. Lopata, J. Phys. Chem. Lett. 8, 3991 (2017).

${ }^{39}$ A. Bruner, S. Hernandez, A. S. Folorunso, A. Sissay, F. Mauger, P. M. Abanador, R. R. Jones, L. F. DiMauro, M. Gaarde, M. B. Gaarde, K. J. Schafer, and K. Lopata, "Molecular modes of charge migration" (unpublished).

${ }^{40}$ H. G. Muller, Appl. Phys. B: Lasers Opt. 74, s17 (2002).

${ }^{41}$ X. Andrade, D. Strubbe, U. De Giovannini, A. Larsen, M. Oliveira, J. AlberdiRodriguez, A. Varas, I. Theophilou, N. Helbig, M. Verstraete, L. Stella, F. Nogueira, A. Aspuru-Guzik, A. Castro, M. Marques, and A. Rubio, Phys. Chem. Chem. Phys. 17, 31371 (2015).
${ }^{42}$ C. Legrand, E. Suraud, and P.-G. Reinhard, J. Phys. B: At., Mol. Opt. Phys. 35, 1115 (2002).

${ }^{43}$ S. Lias, "Ionization energy evaluation," in NIST Chemistry WebBook, NIST Standard Reference Database Number 69, edited by P. Linstrom and W. Mallard (National Institute of Standards and Technology, Gaithersburg, MD, 2018), retrieved December 10, 2018.

${ }^{44}$ M. Lewenstein, P. Saliéres, and A. L'Huillier, Phys. Rev. A 52, 4747 (1995).

${ }^{45}$ M. Bellini, C. Lyngå, A. Tozzi, M. B. Gaarde, T. W. Hänsch, A. L’Huillier, and C.-G. Wahlström, Phys. Rev. Lett. 81, 297 (1998).

${ }^{46}$ Y. Mairesse, A. de Bohan, L. Frasinski, H. Merdji, L. Dinu, P. Monchicourt, P. Breger, M. Kovačev, R. Taïeb, B. Carré, H. Muller, P. Agostini, and P. Salières, Science 302, 1540 (2003).

${ }^{47}$ P. Antoine, A. L'Huillier, and M. Lewenstein, Phys. Rev. Lett. 77, 1234 (1996).

${ }^{48}$ M. Frolov, N. Manakov, T. Sarantseva, and A. Starace, J. Phys. B: At., Mol. Opt. Phys. 42, 035601 (2009).

${ }^{49}$ R. Mulliken, J. Chem. Phys. 3, 720 (1935).

${ }^{50}$ J. Cooper, Phys. Rev. 128, 681 (1962).

${ }^{51}$ S. B. Schoun, R. Chirla, J. Wheeler, C. Roedig, P. Agostini, L. F. DiMauro, K. J. Schafer, and M. B. Gaarde, Phys. Rev. Lett. 112, 153001 (2014).

${ }^{52}$ H. J. Wörner, H. Niikura, J. B. Bertrand, P. B. Corkum, and D. M. Villeneuve, Phys. Rev. Lett. 102, 103901 (2009).

${ }^{53}$ C. E. Brion and K. H. Tan, Chem. Phys. 34, 141 (1978).

${ }^{54}$ A. Hitchcock, C. Brion, and M. van der Wiel, Chem. Phys. 45, 461 (1980).

${ }^{55}$ For the consistent decrease in GD for $\mathrm{N}_{2} \mathrm{O}$ near the cut off, this feature's energetic position was found to scale with the laser intensity, indicating that it is not a structural feature that fits within the scope of this paper.

${ }^{56}$ F. Mauger, P. M. Abanador, T. D. Scarborough, T. T. Gorman, P. Agostini, L. F. DiMauro, K. Lopata, K. J. Schafer, and M. B. Gaarde, "High-harmonic spectroscopy of transient two-center interference calculated with time-dependent density-functional theory" (unpublished).

${ }^{57}$ P. Sándor, A. Sissay, F. Mauger, P. M. Abanador, T. T. Gorman, T. D. Scarborough, M. B. Gaarde, K. Lopata, K. J. Schafer, and R. R. Jones, Phys. Rev. A 98, 043425 (2018). 